\title{
DETERMINATION OF THE SIX DEGREES OF FREEDOM OF A MOVING CIVIL-ENGINEERING VEHICLE
}

\author{
Jean-François LE CORRE and Gaëtan GARCIA \\ Laboratoire d'Automatique de Nantes \\ ENSM. 1 rue de la Noë, 44072 Nantes Cedex 03.
}

\section{SUMMARY}

This paper presents a new system (SIREM) capable of computing the six degrees of freedom and the speed of a moving vehicle. It uses a rotating linear CCD camera on board the vehicle and light sources positioned with precision on the site. The main characteristics of the sensor are reviewed and the equations and method used to compute the position and attitude of the vehicle are presented.

\section{INTRODUCTION}

To automate the work of a civil-engineering vehicle, it is necessary to control the position of its tool. This tool is fixed on the vehicle via actuators, in such a way that it is able to perform a task and meet the requirements of the performance specifications of on-going work.

The tool is controlled in a frame fixed to the vehicle, which means that controlling the tool requires the position and attitude of the vehicle in the absolute reference frame of the job-site. Hence, measuring the position and attitude is a prerequisite to automation.

The sensory system SIREM can supply information on the position and attitude of civil-engineering equipment. SIREM (Système Intelligent de Repérage d'Engins Mobiles: Intelligent System for the Localization of Moving Vehicles) is capable of providing at any instant the six degrees of freedom of the mobile : the three coordinates $\mathrm{x}, \mathrm{y}, \mathrm{z}$ and the three attitude angles (pitch, roll and yaw) in the absolute reference frame. Moreover, SIREM yields accurate results $\left(10 \mathrm{~cm}\right.$ on the position and $0,1^{\circ}$ on the angles) for a mobile moving at $10 \mathrm{~cm}$ per minute.

In this paper, we present the principle of the sensor, its technical characteristics and a method used to compute the coordinates $(x, y, z)$ and the attitude (pitch, roll, yaw) of the vehicle in the job-site frame.

\section{PRINCIPLE OF SIREM}

Punctual light sources (beacons) are allocated on the job-site. The position of every beacon is known in the job-site frame. SIREM is a sensor located on board the vehicle. It is able to measure, in its own frame, the azimuth and height of the beacon that is read at a given instant (figure 1). 
When several beacons have been read, SIREM computes the coordinates, the attitude angles and the speed of the vehicle in the job-site frame. In practice, the data from the last beacon read is added to previous measures, so that the coordinates, attitude and speed are computed at each reading. Thus, the tool of the vehicle can be controlled automatically for the task to be achieved.

SIREM is composed of a hardware part and a software part.

The hardware is composed of:

- a linear camera (50 mm lens, vertical linear CCD sensor with 1728 pixels) mounted on a rotating board, actuated by a motor. The rotation axis is equipped with an incremental encoder and turning contacts.

- a micro-computer (Intel 80286, $12 \mathrm{MHz}$, mathematic coprocessor) equipped with a digitization card for the camera, Input/Output cards, a counter card for the incremental encoder and power and control circuits for the motor.

When the linear detector is lighted by a beacon, the height angle $(\sigma)$ is measured by the linear camera whereas the azimuth angle $(\lambda)$ is measured by the incremental encoder (figure 2).

The software part contains the following elements :

- a real-time executive (RTC), programmed in C.

- a supervisor task, which manages three other tasks : data acquisition, data filtering and position and attitude computation.

The first two tasks provide information to the position and attitude computation module, at every reading of a beacon, namely : the values $\lambda$ and $\sigma$, the number $i$ of the beacon and the time instant $t_{i}$ of the reading.

The computation task uses the data from the readings and the positions of the beacons in the job-site frame to compute the position and attitude of the vehicle in the job-site frame at the time instant of the last reading.

\section{The main present characteristics of SIREM are :}

distance from the vehicle to the beacons : 3 to 45 meters.

maximum speed of the vehicle : 10 meters per minute.

rotation speed of the sensor : from 1 round in 2 seconds to 1 round in 20 seconds.

integration time of the camera : $1,210^{-3}$ second.

vertical image angle : $20^{\circ}$.

precision on $\sigma$ and $\lambda: 0,05^{\circ}$ and $0,1^{\circ}$ respectively.

precision on the position of the vehicle $: 10 \mathrm{~cm}$ on $\mathrm{x}$ and $\mathrm{y}, 5 \mathrm{~cm}$ on $\mathrm{z}$.

precision on the attitude of the vehicle : $0,1^{\circ}$ on the pitch, roll and yaw. 


\section{the computation module of SIREM :}

Several methods have been developed or are under development for the computation of the position and attitude of the vehicle using the measures :

- static localization for a motionless vehicle.

- dynamic localization for a moving vehicle. In this case, two approaches have been used : deterministic and stochastic (using and extended Kalmann filtering). For each of these two approaches, two cases have been considered :

- the two-dimensional case : the vehicle moves in a plane [LE CORRE 91].

- the three-dimensional case : the running surface is partially known.

The method we propose in this paper deals with the three-dimensional case, with a deterministic approach.

\section{DETERMINISTIC DYNAMIC 3D LOCALIZATION}

\subsection{Notations}

We define the following frames (figure 3):

$\mathrm{O}:$ absolute reference frame or job-site frame ;

$\mathrm{M}_{\mathrm{i}}$ : the frame of the mobile at time $\mathrm{t}_{\mathrm{i}}$.

$\mathrm{C}_{\mathrm{i}}$ : the sensor frame at time $\mathrm{t}_{\mathrm{i}}$.

B : a frame attached to the beacons.

and the homogeneous transforms :

${ }^{O} \mathrm{~T}_{\mathrm{Mi}}$ : describes the position and attitude of the mobile in the absolute reference frame $\mathrm{O}$ at time $\mathrm{t}_{\mathrm{i}}$. It is the transform we wish to compute and contains six independent values.

$\operatorname{MiT}_{\mathrm{Ci}}$ : the position of the sensor frame with respect to the vehicle. Although the subscript appears for consistency with previous definitions, this transform is a (known) constant since the sensor is fixed on the vehicle. Hence, ${ }^{M i} T_{C i}={ }^{M} T_{C}$.

${ }^{C i} \mathrm{~T}_{\mathrm{B}}$ : describes the position of the read beacons in the sensor frame.

We can write the following matrix equation (see figure 3) :

$$
\begin{aligned}
& { }^{\mathrm{O}} \mathrm{T}_{\mathrm{Mi}}={ }^{\mathrm{O}} \mathrm{T}_{\mathrm{B}} * \mathrm{~B}_{\mathrm{Ci}} * \mathrm{Ci}_{\mathrm{Mi}} \\
& \text { with }{ }^{\mathrm{B}} \mathrm{T}_{\mathrm{Ci}}=\left[{ }^{\mathrm{Ci}} \mathrm{T}_{\mathrm{B}}\right]^{-1}, \text { which is easy to calculate. }
\end{aligned}
$$

At time $\mathrm{t}_{\mathrm{i}}$, beacon $\mathrm{B}_{\mathrm{i}}$ is read (this convention can be used by renumbering the beacons if required).

Let $\left[{ }^{k} x_{i},{ }^{y_{i}},{ }^{z_{i}}\right]$ be the coordinates of beacon $B_{i}$ in the frame $C_{k}$. 
In $\mathrm{C}_{\mathrm{i}}$, it is easy to write a relation between the azimuth and height and the coordinates of the beacon:

$$
\tan \lambda_{i}=\frac{{ }^{i} x_{i}}{{ }^{i} y_{i}} \quad \tan \sigma_{i}=\frac{{ }^{i} z_{i}}{\sqrt{i_{x_{i}}^{2}+{ }^{i} y_{i}^{2}}}
$$

\subsection{Formulation of the problem}

The computation of the position and attitude of the vehicle at instant $t_{i}$ requires to integrate the equations of the movement on the running surface. This implies a model of the surface and a law for the movement.

We shall here consider the case where the following properties, which will be later justified, are locally satisfied :

- the surface can be approximated by its tangent plane ;

- the movement of the vehicle is piecewise linear ;

- its speed is piecewise constant.

Let $b_{i}$ be the distance from the origin of frame $C_{i}$ to beacon $B_{i}$. In the conditions formulated before, we can write (figure 4) :

$$
\begin{aligned}
& { }^{0} \mathrm{x}_{\mathrm{i}}-\mathrm{v} \cdot\left(\mathrm{t}_{\mathrm{k}}-\mathrm{t}_{0}\right)=\mathrm{b}_{\mathrm{i}} \cdot \cos \sigma_{\mathrm{i}} \cdot \cos \lambda_{\mathrm{i}} \\
& { }^{0} \mathrm{y}_{\mathrm{i}}=\mathrm{b}_{\mathrm{i}} \cdot \cos \sigma_{\mathrm{i}} \cdot \sin \lambda_{\mathrm{i}} \\
& { }^{0} \mathrm{z}_{\mathrm{i}}=\mathrm{b}_{\mathrm{i}} \cdot \sin \sigma_{\mathrm{i}}
\end{aligned}
$$

The computation of the coordinates will be achieved as soon as $v$ and $b_{i}$ are found.

At time $t_{j}$, the system reads a second beacon $B_{j}$. This yields three equations for the coordinates of $B_{j}$ in the sensor frame at time $t_{0}$.

It is then possible to consider the distance $d_{i j}$ between $B_{i}$ and $B_{j}$, which is the same in any frame.

$$
\mathrm{d}_{\mathrm{ij}}{ }^{2}=\left(0 \mathrm{x}_{\mathrm{i}}-{ }^{0} \mathrm{x}_{\mathrm{j}}\right)^{2}+\left({ }^{0} \mathrm{y}_{\mathrm{i}}-{ }^{0} \mathrm{y}_{\mathrm{j}}\right)^{2}+\left(\mathrm{O}_{\mathrm{i}}-{ }^{0} \mathrm{z}_{\mathrm{j}}\right)^{2}
$$

The three terms can be written as :

$$
\begin{aligned}
& \left({ }^{0} x_{i}-{ }^{0} x_{j}\right)^{2}=v^{2} \Delta t_{i j}{ }^{2}+b_{i}{ }^{2} c c_{i}{ }^{2}+b_{j}{ }^{2} c c_{j}{ }^{2}-2 b_{i} b_{j} c c_{i} c c_{j}+2 v \cdot \Delta t_{i j} \cdot\left(b_{i} c c_{i}-b_{j} c c_{j}\right) \\
& \left({ }^{0} \mathrm{y}_{\mathrm{i}}-{ }^{0} \mathrm{y}_{\mathrm{j}}\right)^{2}=\mathrm{b}_{\mathrm{i}}{ }^{2} \mathrm{cs}_{\mathrm{i}}{ }^{2}+\mathrm{b}_{\mathrm{j}}{ }^{2} \mathrm{cs}_{\mathrm{j}}^{2}-2 \mathrm{~b}_{\mathrm{i}} \mathrm{b}_{\mathrm{j}} \mathrm{cs} \mathrm{scs}_{\mathrm{j}} \\
& \left({ }^{0} \mathrm{z}_{\mathrm{i}}-{ }^{0} \mathrm{z}_{\mathrm{j}}\right)^{2}=\mathrm{b}_{\mathrm{i}}{ }^{2} \mathrm{~s}_{\mathrm{i}}^{2}+\mathrm{b}_{\mathrm{j}}{ }^{2} \mathrm{sj}^{2}-2 \mathrm{~b}_{\mathrm{i}} \mathrm{b}_{\mathrm{j}} \mathrm{Si}_{\mathrm{j}}
\end{aligned}
$$

with :

$$
\begin{aligned}
& \mathrm{cc}_{\mathrm{i}}=\cos \left(\sigma_{\mathrm{i}}\right) \cdot \cos \left(\lambda_{\mathrm{i}}\right) \\
& \mathrm{cs}_{\mathrm{i}}=\cos \left(\sigma_{\mathrm{i}}\right) \cdot \sin \left(\lambda_{\mathrm{i}}\right) \\
& \mathrm{s}_{\mathrm{i}}=\sin \left(\sigma_{\mathrm{i}}\right)
\end{aligned}
$$


We thus obtain the equation :

$$
\begin{array}{ll} 
& f(i, j)=b_{i}{ }^{2}+b_{j}^{2}-\alpha_{i j} \cdot b_{i} \cdot b_{j}-d_{i j}{ }^{2}+t_{i j}^{2} \cdot v^{2}+t_{i j} \cdot \beta_{i} \cdot v \cdot b_{i}-t_{i j} \cdot \beta_{j} \cdot v \cdot b_{j}=0 \\
\text { with : } \quad & \alpha_{i j}=2 \cdot\left[c c_{i} \cdot c c_{j}+c s_{i} \cdot c s_{j}+s_{i} \cdot s_{j}\right] \\
& \beta_{i}=2 \cdot c c_{i} \\
& t_{i j}=t_{j}-t_{i}
\end{array}
$$

Our problem is then equivalent to computing the values $v, b_{i}$ and $b_{j}$. Two readings yield only one equation. The $\mathrm{n}^{\text {th }}$ reading will add one extra equation and allow to write $\mathrm{n}-1$ new equations. Hence, with four readings, we get 5 unknowns ( $\mathrm{v}$ and the distances to four beacons) and 6 equations. We shall then use four readings and solve a redundant non-linear system in five unknowns.

\subsection{Solution of the system}

We have six non-linear equations in five unknowns, which we can write as :

$$
\begin{aligned}
& f(i, j)=f\left(v, b_{i}, b_{j}\right)=0 \\
& \text { for } j=1,2,3 \text { and } i<j
\end{aligned}
$$

We shall present two methods to solve this system. Both derive from the NewtonRaphson method. The first takes advantage of the special form of the Jacobian matrix of the system. The second decomposes the set of equations into two sub-systems.

The Jacobian matrix $J$ of the system $f\left(v, b_{i}, b_{j}\right)=0$ contains 30 terms, of which 18 are a priori different from 0 .

$$
\begin{aligned}
& J_{V}(i, j)=\frac{\delta f(i, j)}{\delta v}, \quad J_{b}(k, i, j)=\frac{\delta f(i, j)}{\delta b_{k}} \text { with } k=i \text { or } k=j \\
& \mathrm{~J}=\left[\begin{array}{ccccc}
\mathrm{J}_{\mathrm{V}}(0,1) & \mathrm{J}_{\mathrm{b}}(0,0,1) & \mathrm{J}_{\mathrm{b}}(1,0,1) & 0 & 0 \\
\mathrm{~J}_{\mathrm{V}}(0,2) & \mathrm{J}_{\mathrm{b}}(0,0,2) & 0 & \mathrm{~J}_{\mathrm{b}}(2,0,2) & 0 \\
\mathrm{~J}_{\mathrm{V}}(0,3) & \mathrm{J}_{\mathrm{b}}(0,0,3) & 0 & 0 & \mathrm{~J}_{\mathrm{b}}(3,0,3) \\
\mathrm{J}_{\mathrm{V}}(1,2) & 0 & \mathrm{~J}_{\mathrm{b}}(1,1,2) & \mathrm{J}_{\mathrm{b}}(2,1,2) & 0 \\
\mathrm{~J}_{\mathrm{V}}(1,3) & 0 & \mathrm{~J}_{\mathrm{b}}(1,1,3) & 0 & \mathrm{~J}_{\mathrm{b}}(3,1,3) \\
\mathrm{J}_{\mathrm{V}}(2,3) & 0 & 0 & \mathrm{~J}_{\mathrm{b}}(2,2,3) & \mathrm{J}_{\mathrm{b}}(3,2,3)
\end{array}\right]
\end{aligned}
$$

The terms in $J$ can be expressed as functions of the measures $\lambda_{i}, \sigma_{i}, t_{i}$.

$$
\begin{aligned}
& \mathrm{J}_{\mathrm{v}}(\mathrm{i}, \mathrm{j})=2 \cdot \mathrm{t}_{\mathrm{ij}}{ }^{2} \cdot \mathrm{v}+\mathrm{t}_{\mathrm{ij}} \cdot \beta_{\mathrm{i}} \cdot \mathrm{b}_{\mathrm{i}}-\mathrm{t}_{\mathrm{ij}} \cdot \beta_{\mathrm{j}} \cdot \mathrm{b}_{\mathrm{j}} \\
& \mathrm{J}_{\mathrm{b}}(\mathrm{i}, \mathrm{i}, \mathrm{j})=2 \cdot \mathrm{b}_{\mathrm{i}}-\alpha_{\mathrm{ij}} \cdot \mathrm{b}_{\mathrm{j}}+\mathrm{t}_{\mathrm{ij}} \cdot \beta_{\mathrm{i} \cdot \mathrm{v}} \\
& \mathrm{J}_{\mathrm{b}}(\mathrm{j}, \mathrm{i}, \mathrm{j}, \mathrm{j})=2 \cdot \mathrm{b}_{\mathrm{j}}-\alpha_{\mathrm{ij}} \cdot \mathrm{b}_{\mathrm{i}}-\mathrm{t}_{\mathrm{j} j} \cdot \beta_{\mathrm{j}} \cdot \mathrm{v}
\end{aligned}
$$




\section{First method.}

It solves the non-linear, redundant system globally using the special form of the jacobian matrix.

The Jacobian matrix has the following form : $J=\left[\begin{array}{ll}A & B \\ C & D\end{array}\right]$

where $\mathrm{A}$ is a full $3 \times 2$ matrix, $\mathrm{B}$ is diagonal $3 \times 3, \mathrm{C}$ is $3 \times 2$ with one single column different from zero and $\mathrm{D}$ is an anti-diagonal matrix.

and the system to be solved is : $\left[\begin{array}{cc}A & B \\ C & D\end{array}\right]\left[\begin{array}{c}d v \\ d b_{0} \\ d b_{1} \\ d_{2} \\ d b_{3}\end{array}\right]=-\left[\begin{array}{c}f(0,1) \\ f(0,2) \\ f(0,3) \\ f(1,2) \\ f(1,3) \\ f(2,3)\end{array}\right]$

Let $X_{1}=\left[\begin{array}{c}d v \\ d b_{0}\end{array}\right], X_{2}=\left[\begin{array}{l}d b_{1} \\ d b_{2} \\ d b_{3}\end{array}\right], F_{1}=\left[\begin{array}{c}f(0,1) \\ f(0,2) \\ f(0,3)\end{array}\right] F_{2}=\left[\begin{array}{c}f(1,2) \\ f(1,3) \\ f(2,3)\end{array}\right]$

We have two matrix equations :

$$
\begin{aligned}
& \text { A X } X_{1}+\mathrm{BX}_{2}=-\mathrm{F}_{1} \\
& \mathrm{C} \mathrm{X}+\mathrm{DX}_{2}=-\mathrm{F}_{2}
\end{aligned}
$$

Since $B$ is easy to invert, (7) gives $X_{2}$ as a function of $X_{1}: X_{2}=-B^{-1} F_{1}-B^{-1} A X_{1}$.

This expression is then substituted to $X_{2}$ in (8). We get an over-determined system of the form :

$$
\mathrm{MX} \mathrm{X}_{1}=\mathrm{C}_{1}
$$

which is solved by a Least-Square Method, i.e. :

$$
X_{1}=\left(M^{\top} M\right)^{-1} M^{T} C_{1} .
$$

This gives $X_{1}$ and $X_{2}$, which are the increments to add to the unknowns $v, b_{0}, \ldots, b_{4}$ in the Newton-Raphson method. These computations must be iterated until convergence is reached. 


\section{Second method}

principle :

This second method is a generalization of the solution presented in [LE CORRE 90] to solve the static problem. Indeed, if $\mathrm{v}$ is set to zero in system (4) we get the equations of the static problem. It has been shown in [DEVISE 90] that this method was more efficient than general methods of mathematical libraries.

In this paragraph, we only show how to manipulate the system in such a way that the solution is found by solving repeatedly a (simple) $3 \times 3$ non-linear system and an overdetermined non-linear $3 \times 2$ system. The latter can be transformed into a non-linear $2 \times 2$ system, which is easily solved using a Newton-Raphson method.

The idea consists of the following steps :

1- initialize $v$ and $b_{0}$ with values that are supposed to be close to the solution ;

2 - introduce these values in the first three equations $f(i, j)=0$ and calculate the corresponding values of $b_{1}, b_{2}$ and $b_{3}$;

3 - enter $b_{1}, b_{2}$ and $b_{3}$ into the last three functions $f(i, j)$ and check wether they are equal to zero or not. If not, compute variations of $v$ and $b_{0}$ that make $v$ and $b_{0}$ best satisfy the system (i.e. in the Least-Squares sense) and return to step 2.

\section{solution of the overdetermined non-linear system}

Solving the system $f(i, j)=0$ with $j=2,3$ and $i<j$ for given values of $v$ and $b_{0}$ requires to calculate the jacobian matrix of the sub-system.

$$
\begin{aligned}
& \operatorname{df}(1,2)=\frac{\delta g}{\delta v} \cdot d v+\frac{\delta g}{\delta b_{0}} \cdot d b_{0} \\
& \operatorname{df}(1,3)=\frac{\delta h}{\delta v} \cdot d v+\frac{\delta h}{\delta b_{0}} \cdot d b_{0} \\
& \operatorname{df}(2,3)=\frac{\delta l}{\delta v} \cdot d v+\frac{\delta l}{\delta b_{0}} \cdot d b_{0}
\end{aligned}
$$

with :

$$
\begin{aligned}
& \mathrm{df}(1,2)=J_{V}(1,2) \cdot d v+J_{b}(1,1,2) \cdot d b_{1}+j \mathrm{j}(2,1,2) \cdot d b_{2} \\
& \mathrm{df}(1,3)=J_{\mathrm{V}}(1,3) \cdot d v+J_{b}(1,1,3) \cdot d b_{1}+j \mathrm{jb}(3,1,3) \cdot d b_{3} \\
& \mathrm{df}(2,3)=J_{V}(2,3) \cdot d v+J_{b}(1,2,3) \cdot d b_{2}+j \mathrm{jb}(3,1,3) \cdot d b_{3}
\end{aligned}
$$

$d b_{1}, d b_{2}$ and $d b_{3}$ are calculated as functions of $d v$ and $d b_{0}$ by differentiating the first three equations of system (4). We obtain : 


$$
\left[\begin{array}{ccc}
\mathrm{J}_{\mathrm{b}}(1,0,1) & 0 & 0 \\
0 & \mathrm{Jb}_{\mathrm{b}}(2,0,2) & 0 \\
0 & 0 & \mathrm{~J}_{\mathrm{b}}(3,0,3)
\end{array}\right] \times\left[\begin{array}{l}
\mathrm{db}_{1} \\
\mathrm{db}_{2} \\
\mathrm{db}_{3}
\end{array}\right]=\left[\begin{array}{c}
\mathrm{c}_{1} \\
\mathrm{c}_{2} \\
\mathrm{c}_{3}
\end{array}\right]
$$

with :

$$
\begin{aligned}
& c_{1}=-\left[J_{V}(0,1) \cdot d v+J_{b}(0,0,1) \cdot d b_{0}\right] \\
& c_{2}=-\left[J_{V}(0,2) \cdot d v+J_{b}(0,0,2) \cdot d b_{0}\right] \\
& c_{3}=-\left[J_{V}(0,3) \cdot d v+J_{b}(0,0,3) \cdot d b_{0}\right]
\end{aligned}
$$

The system is diagonal, so the calculation of $\mathrm{db}_{1}, \mathrm{db}_{2}$ and $\mathrm{db}_{3}$ is obvious.

After computing the values $\mathrm{v}$ and $\mathrm{b}_{\mathrm{i}}$ using the first or the second method, the homogeneous transform ${ }^{\mathrm{C}} \mathrm{T}_{\mathrm{B}}$, which represents the position of the beacons in the sensor frame, can be calculated. Hence, using equation (1) yields the homogeneous transform ${ }^{O} T_{M}$ that defines the position and attitude of the vehicle in the job-site frame.

\section{CONCLUSION}

In this paper, we have first presented the principle and the characteristics of SIREM, together with a dynamic localization method.

The principle of SIREM uses a three-dimensional dynamic localization sensor. It has been designed by the Laboratoire Central des Ponts et Chaussées to meet the requirements of automation of civil-engineering equipment [PEYRET 89]. The hardware has been realized by CYBERG. The computation and the localization software have been developed by the Control Laboratory of the Ecole Nationale Supérieure de Mécanique in Nantes. Job-site tests are scheduled for 1991-1992 at the Laboratoire Central des Ponts et Chaussées, in cooperation with the road manufacturer Entreprise Jean Lefebvre.

We have then presented a method for the dynamic localization of a vehicle (six degrees of freedom) based on a deterministic approach. At each reading of a beacon, i.e. at an average rate of one every two thirds of a second, the computation yields the position, attitude and speed of the vehicle on a partially known running surface. In addition to the computation of the position and attitude of the mobile, our method gives information to build a model of the surface, since it position and normal are given every third of a meter for a speed of ten meters per minute.

This work is supported by the the French Ministry of Research and Technology in the framework of a two-year contract, with the partners presented in the previous paragraphs. 


\section{REFERENCES}

[LE CORRE 90] J.F. LE CORRE and F. PEYRET, "SIREM : the absolute location of civil-engineering equipment", Mechatronics Systems Engineering 1, 1990, Kluwer Academic Publishers, Netherlands.

[PEYRET 89] F. PEYRET, "La localisation absolue des engins routiers - Introduction au projet SIREM", Rapport de recherche interne du Laboratoire Centrale des Ponts et Chaussées, Nantes, janvier 1989.

[DEVISE 90] O. DEVISE, "Localisation dynamique de robots mobiles", DEA d'Automatique, Informatique Appliquée et Systèmes Electroniques, Université de Nantes, 1990.

[LE CORRE 91] J.F. LE CORRE and G. GARCIA, "Dynamic localization of a vehicleusing passive beacons", Second France-Israël Symposium on Robotics, Saclay, 810 april 1991.

\section{FIGURES}

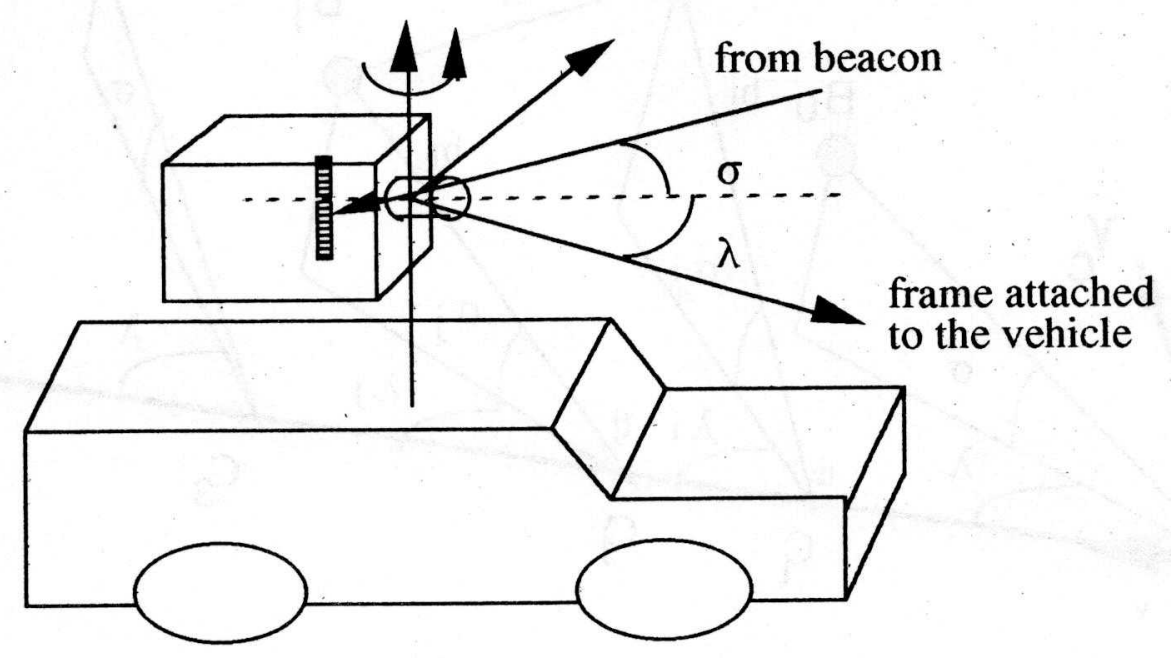

figure 1. The vehicle and its sensor

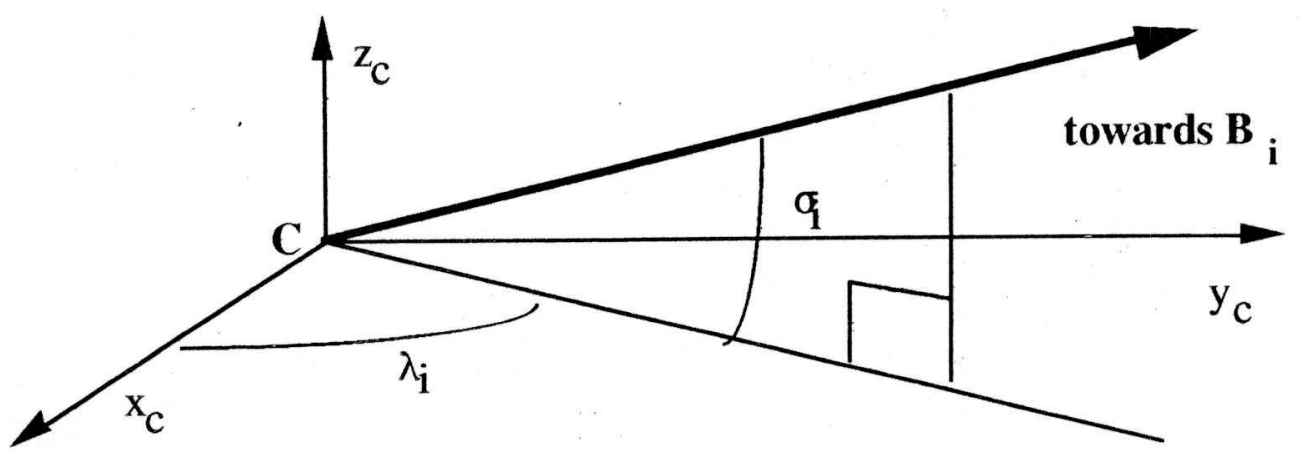

figure 2 . The sensor frame and the measures 


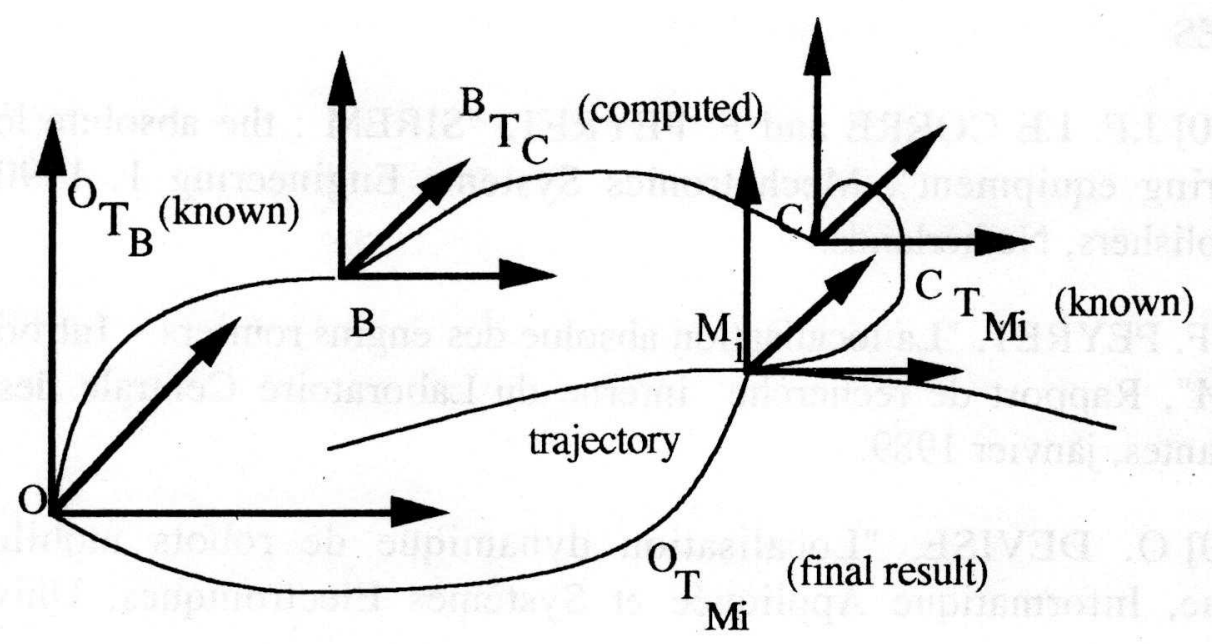

figure 3. The frames used in the localization method

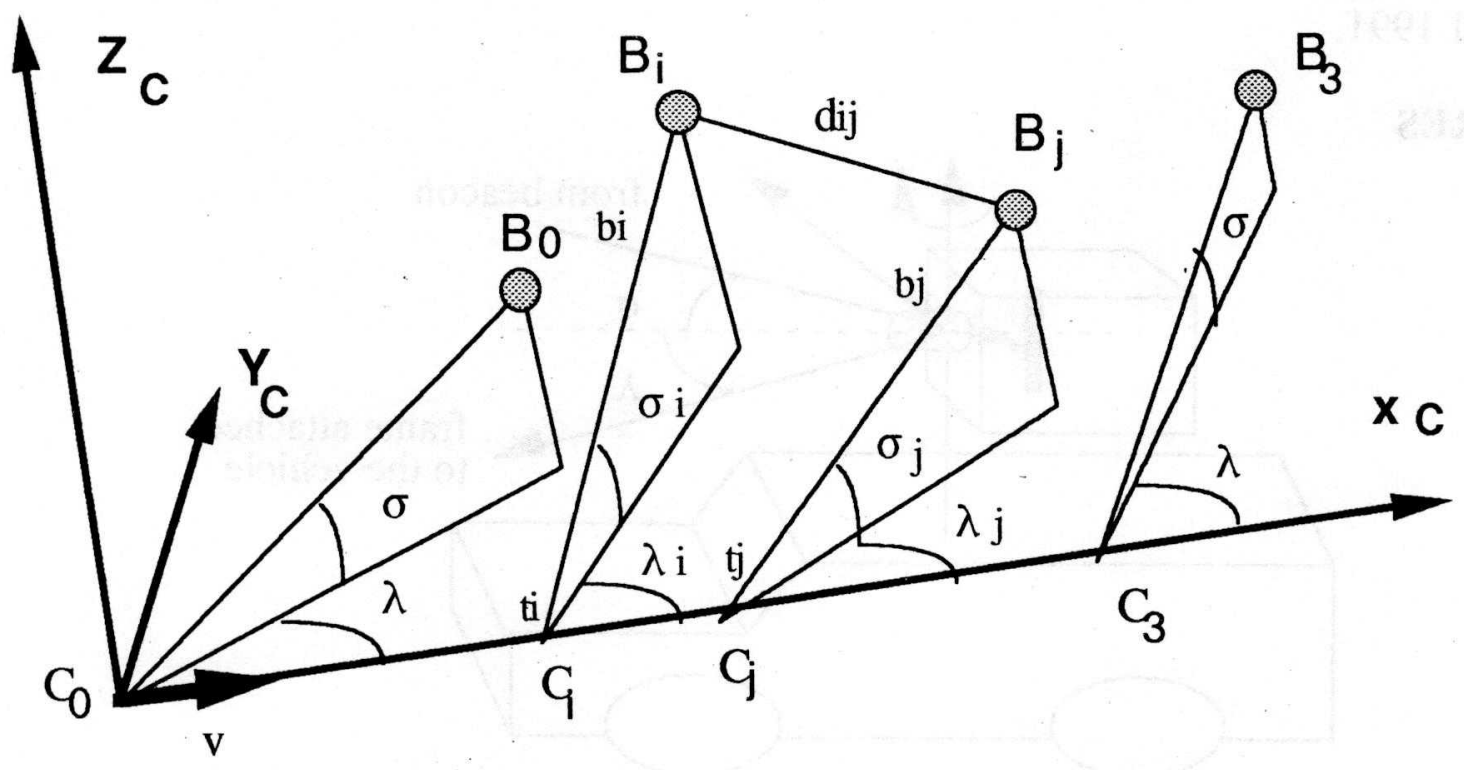

figure 4

The movement of the vehicle and the measures 